\title{
KERNEL FUNCTIONS ON DOMAINS WITH HYPERELLIPTIC DOUBLE $\left({ }^{1}\right)$
}

BY

\author{
WILLIAM H. BARKER II
}

\begin{abstract}
In this paper we show that the structure of the Bergman and Szegö kernel functions is especially simple on domains with hyperelliptic double. Each such domain is conformally equivalent to the exterior of a system of slits taken from the real axis, and on such domains the Bergman kernel function and its adjoint are essentially the same, while the Szegö kernel function and its adjoint are elementary and can be written in a closed form involving nothing worse than fourth roots of polynomials. Additionally, a number of applications of these results are obtained.
\end{abstract}

Suppose that $थ$ is a compact Riemann surface and that $f$ is a meromorphic function on $\mathcal{W}$. The function $f$ is then an analytic mapping onto the Riemann sphere $S_{0}$, and it is a fundamental fact that each point of $S_{0}$ is assumed precisely the same number of times. This common number is called the order of $f$. Thus, for example, if the order of $f$ is one, then W is a conformal homeomorph of the Riemann sphere. The next possibility is for W to admit a meromorphic function of order two, and in this case $\mathcal{W}$ is termed hyperelliptic. The hyperelliptic surfaces are thus in this sense the second simplest class of Riemann surfaces, and it is widely recognized that the hyperelliptic surfaces play a significant role in the more general theory of compact Riemann surfaces. Observe, also, that all Riemann surfaces of genus zero, one, or two are hyperelliptic.

Our concern here, however, is not with compact Riemann surfaces per se, but rather with plane domains. The significance for us of the compact surfaces is that each plane domain of connectivity $n$ can be embedded in a natural way in a compact Riemann surface of genus $n-1$ called the double of the domain. See, for example, [13] or [12].

Definition 1. For each $n \geqslant 1$, let $\mathbb{Q}_{n}$ be the class of plane domains whose boundary is formed by $n$ disjoint peicewise analytic curves. We shall denote by $H_{n}$ the class of all domains in $\mathbb{Q}_{n}$ which possess a hyperelliptic double, by $\delta_{n}$ the class of all domains in $\mathbb{Q}_{n}$ which are symmetric with respect to the real

Received by the editors January 28, 1976.

AMS (MOS) subject classifications (1970). Primary 30A31; Secondary 30A24, 30A42, 30A46.

Key words and phrases. Hyperelliptic double, kernel function, plane domains.

(')This work forms part of the author's Doctoral Dissertation at Stanford University written under the direction of M. M. Schiffer. 
axis and whose intersection with the open upper half plane is simply connected, and by $\Sigma_{n}$ the class of all domains which are the exterior of a system of $n$ slits taken from the real axis.

Our concern now is to indicate how the special structure of the compact hyperelliptic Riemann surfaces is reflected in the class $H_{n}$. We will focus here on the structure of the Bergman and Szegö kernel functions defined on domains in $H_{n}$. The tools developed here will enable an elegant treatment of Noether's theorem for domains in $H_{n}$, as well as the variational theory of functionals on domains in $H_{n}$.

We begin with the fundamental geometric fact concerning domains in the class $H_{n}$. The proof of this result is immediate from well-known properties of hyperelliptic surfaces. See [3].

THEOREM 1. The following are equivalent:

(1) $D \in H_{n}$,

(2) $D$ can be mapped one-to-one conformally onto a domain in $\Sigma_{n}$,

(3) $D$ admits an anticonformal involution possessing precisely $2 n$ fixed points on the boundary of $D$.

Theorem 1 is important in that the class $\Sigma_{n}$ will play a role here similar to that played by the unit disk in the theory of functions on simply connected domains. Indeed, it will become evident that domains in the class $\Sigma_{n}$ are in many respects the second simplest class after the unit disk. As a consequence of Theorem 1, observe also that $H_{n} \supseteq \delta_{n} \supseteq \Sigma_{n}$.

Definition 2. Let $r_{1}, r_{2}, \ldots, r_{2 n}$ be the endpoints of the line segments which form the boundary of $D \in \Sigma_{n}$. Then the "structure polynomial" of $D$ is defined to be the polynomial $q(z)=\Pi\left(z-r_{j}\right)$ where the product is taken over all finite endpoints $r_{i}$.

Note that if $D \in \Sigma_{n}$ has the structure polynomial $q(z)$, then the double of $D$ is conformally equivalent to the Riemann surface $w^{2}-q(z)=0$. As an immediate consequence of this, let $e_{1}, e_{2}, \ldots, e_{2 n}$ be $2 n$ distinct points located in the finite plane. Then a necessary and sufficient condition for the Riemann surface $w^{2}-\Pi_{j=1}^{2 n}\left(z-e_{j}\right)=0$ to be the double of a plane domain is for the $e_{j}$ to be located in a circle. Compare, for example, [6].

Now let $D$ be a domain in the class $\delta_{n}$ and suppose that $\omega_{\mu}$ is the harmonic measure on $D$ with boundary values $\delta_{\mu \nu}$ on $\gamma_{\nu}$. Each $\omega_{\mu}$ is the real part of a multivalued analytic function $w_{\mu}(z)=\omega_{\mu}(z)+i \sigma_{\mu}(z)$ whose derivative is single-valued on $D$. Observe that because of the symmetry of $D$,

$$
w_{\mu}^{\prime}(z)=\overline{w_{\mu}^{\prime}(\bar{z})}, \quad z \in D, D \in \mathcal{S}_{n} .
$$

We also define the induction coefficients $P_{\mu \nu}, \mu, \nu=0,1,2, \ldots, n-1$, by 


$$
P_{\mu \nu}=-\frac{1}{2 \pi} \oint_{\gamma_{\nu}} \frac{\partial \omega_{\mu}}{\partial n} d s=\frac{1}{2 \pi i} \int_{\gamma_{\nu}} w_{\mu}^{\prime}(z) d z
$$

The matrix $\left[P_{\mu \nu}\right]_{\mu, \nu=1}^{n-1}$ is strictly positive definite and, hence, possesses the inverse matrix $\left[\Pi_{\mu \nu}\right]$.

Continuing with $D \in \mathcal{S}_{n}$, let $K(z, \bar{\zeta})$ and $L(z, \zeta)=(z-\zeta)^{-2} / \pi-l(z, \zeta)$ be the Bergman kernel function and its adjoint for $D$, and let $\tilde{K}(z, \bar{\zeta})$ and $\tilde{L}(z, \zeta)=(z-\zeta)^{-2} / \pi-\tilde{l}(z, \zeta)$ be the semiexact Bergman kernel function and its adjoint for $D$. The kernel functions $K(z, \bar{\zeta})$ and $\tilde{K}(z, \bar{\zeta})$ are analytic in $z$, antianalytic in $\zeta$, and satisfy

$$
\tilde{K}(z, \bar{\zeta})=K(z, \bar{\zeta})-\frac{1}{2 \pi} \sum_{\mu, \nu=1}^{n-1} \Pi_{\mu \nu} w_{\mu}^{\prime}(z) \overline{w_{\nu}^{\prime}(\zeta)}
$$

Similarly,

$$
\tilde{L}(z, \zeta)=L(z, \zeta)-\frac{1}{2 \pi} \sum_{\mu, \nu=1}^{n-1} \Pi_{\mu \nu} w_{\mu}^{\prime}(z) w_{\nu}^{\prime}(\zeta)
$$

Now observe that whenever $\zeta$ is in $D$, so also is $\bar{\zeta}$ in $D$, and so $D$ admits the anticonformal involution $V(\zeta)=\bar{\zeta}$. We thus define the kernel functions

$$
K(z, \zeta)=K(z, \overline{V(\zeta)}) \text { and } \tilde{K}(z, \zeta)=\tilde{K}(z, \overline{V(\zeta)})
$$

The kernel functions $K(z, \zeta)$ and $\tilde{K}(z, \zeta)$ are analytic and symmetric in both variables $z$ and $\zeta$.

Now for $s \in D$, let

$$
\begin{aligned}
& \varphi(t, s)=1 /(t-s)+a_{1}(t-s)+a_{2}(t-s)^{2}+\cdots \\
& \psi(t, s)=1 /(t-s)+c_{1}(t-s)+c_{2}(t-s)^{2}+\cdots
\end{aligned}
$$

be univalent functions on $D$ which map $D$ onto the exterior of a system of, respectively, horizontal and vertical slits. It is known then that

$$
\frac{1}{2 \pi}\left[\varphi^{\prime}(t, s)-\psi^{\prime}(t, s)\right]=\tilde{K}(t, \bar{s}), \quad\left(\varphi^{\prime}(t, s)=\partial \varphi(t, s) / \partial t\right),
$$

and

$$
\frac{1}{2 \pi}\left[\varphi^{\prime}(t, s)+\psi^{\prime}(t, s)\right]=-\tilde{L}(t, s) .
$$

See, for example, [4, p. 82] or [9, pp. 361-368].

Now assume that $s \in D$ is a point on the real axis so that, in particular, $s=\bar{s}=V(s)$. Forming the sum of equations (4) and (5) and using (1), (2), and (3), we obtain, for $s$ real, 


$$
\begin{aligned}
\varphi^{\prime}(t, s) & =-(t-s)^{-2}+a_{1}+2 a_{2}(t-s)^{2}+\cdots \\
& =\pi(K(t, s)-L(t, s)), \\
& =\pi(K(t, s)-L(t, s)), \quad s \text { real in } D .
\end{aligned}
$$

Now let $s$ and $u$ be two points of $D$ taken from the real axis. Then the canonical horizontal slit mappings $\varphi(t, s)$ and $\varphi(t, u)$ satisfy

$$
\varphi(t, u)-\varphi(s, u)=\varphi^{\prime}(u, s) /[\varphi(t, s)-\varphi(u, s)]
$$

and from equation (6) we have

$$
\begin{aligned}
\pi(K(u, s)-L(u, s)) & =\varphi^{\prime}(u, s) \\
& =(\varphi(t, u)-\varphi(s, u))(\varphi(t, s)-\varphi(u, s)) .
\end{aligned}
$$

We also have the power series developments of the functions $\varphi(t, u)$ and $\varphi(t, s)$ near the point $t=s$ :

$$
\begin{gathered}
\varphi(t, u)=\sum_{n=0}^{\infty} b_{n}(t-s)^{n}, \quad b_{n}=\varphi^{(n)}(s, u) / n ! \\
\varphi(t, s)=(t-s)^{-1}+\sum_{n=1}^{\infty} a_{n}(t-s)^{n} .
\end{gathered}
$$

Substituting the expansions (9) and (10) into equation (8), and collecting together terms of equal degree in $(t-s)$, we find that

$$
b_{n}= \begin{cases}\varphi^{\prime}(u, s), & n=1, \\ \varphi(u, s) b_{1}, & n=2, \\ \varphi(u, s) b_{n-1}-\sum_{j=1}^{n-2} a_{j} b_{n-1-j}, & n \geqslant 3 .\end{cases}
$$

Observe that (11) permits us to solve for the $b_{n}$ in terms of the $a_{n}$ and the numbers $\varphi(u, s)$ and $\varphi^{\prime}(u, s)$. Additionally, we have the following important application of the system (11).

THEOREM 2. If $D$ is a domain in the class $\S_{n}$, then for all s and $u$ in $D$,

$$
\frac{\partial^{2}}{\partial s \partial u} \log [K(s, u)-L(s, u)]=2 \pi[K(s, u)-L(s, u)] .
$$

Proof. First let $s$ and $u$ be taken from the real axis in $D$. From equation (6) and the expansion (9), we see that

$$
(\partial / \partial s) \log [K(s, u)-L(s, u)]=2 b_{2} / b_{1}, \quad s \text { and } u \text { real. }
$$

But from (11), the right-hand side of equation (13) is simply $2 \varphi(u, s)$. Thus, 


$$
\frac{\partial^{2}}{\partial s \partial u} \log [K(s, u)-L(s, u)]=2 \varphi^{\prime}(u, s), \quad s \text { and } u \text { real. }
$$

With the restriction that $s$ and $u$ are taken from the real axis, equation (12) follows immediately from equation (6). Observe, however, that both sides of equation (12) are analytic functions in $s$ and $u$, and, consequently, (12) holds for all $s$ and $u$ in $D$.

We remark that identities of the form (12) have been extensively studied in the past by, among others, Schiffer and Hawley [11]. It is interesting to note that the system (11), in concert with equation (6), provides an effective tool for establishing additional, albeit more complicated, identities of this form.

As a final application of equation (6), we have the following result.

THEOREM 3. For $D$ a domain in the class $\Sigma_{n}$, and all $t, s \in D$,

$$
K(t, s)=-l(t, s) \text { and } \tilde{K}(t, s)=-\tilde{l}(t, s) \text {. }
$$

Proof. First note that if $s \in D$ is a point taken from the real axis, then $\varphi(t, s)=1 /(t-s)$. From this fact and equation (6), it follows that (14) is valid for all real $s$ in $D$. But observe that $K(t, s), l(t, s), \tilde{K}(t, s)$, and $\tilde{l}(t, s)$ are all analytic functions of the variable $s$, whence the identity (14) must hold for all $s$ in $D$ as claimed.

For $D$ a domain in the class $\mathbb{Q}_{n}$, let $\hat{K}(z, \bar{\zeta})$ and $\hat{L}(z, \bar{\zeta})$ be the Szegö kernel and its adjoint for $D$. For fixed $\zeta \in D$, the functions $\hat{K}(z, \bar{\zeta})$ and $\hat{L}(z, \zeta)$ are uniquely determined by the conditions:

(a) $\hat{K}(z, \bar{\zeta})$ is analytic for all $z \in D$;

(b) $\hat{L}(z, \zeta)$ is analytic for all $z \in D$ except for a simple pole at $z=\zeta$ with residue $1 / 2 \pi$;

(c) $\hat{L}(z, \zeta) d z=\overline{i \hat{K}(z, \bar{\zeta})}|d z|$ for all $z \in \partial D$.

See, for example, [10].

Now suppose that the domain $D$ is in the class $\Sigma_{n}$ and that the point at infinity is in $D$. The boundary segments of $D$ are then of the form

$$
\gamma_{j}=\left[r_{2 j+1}, r_{2 j+2}\right], \quad j=0,1,2, \ldots, n-1,
$$

and we assume that the endpoints are ordered by the condition $r_{1}<r_{2}$ $<\cdots<r_{2 n}$.

We define two polynomials $q_{1}(z)$ and $q_{2}(z)$ by

$$
q_{1}(z)=\prod_{j=0}^{n-1}\left(z-r_{2 j+1}\right) \text { and } q_{2}(z)=\prod_{j=0}^{n-1}\left(z-r_{2 j+2}\right) ;
$$

the zeros of $q_{1}(z)$ are located at the left-hand endpoints of the boundary segments $\gamma_{j}, j=0,1,2, \ldots, n-1$, while the zeros of $q_{2}(z)$ are located at the right-hand endpoints. The product $q_{1}(z) q_{2}(z)$ is precisely the structure polynomial $q(z)$ for the domain $D$. We further define the function $m(z)$ on $D$ by 


$$
m(z)=q_{2}(z) / q_{1}(z) \text {. }
$$

Observe that $m(z)$ effects an $n$-fold covering of $D$ over the domain

$$
H=\hat{C} \backslash\{w:-\infty \leqslant \operatorname{Re} w \leqslant 0\} .
$$

For $w$ in $H$, we will denote by $\sqrt{w}$ and $\sqrt[4]{w}$ the unique analytic square root and fourth root on $H$ which satisfy $\sqrt{1}=1$ and $\sqrt[4]{1}=1$. Note that

$$
\sqrt{w}=\overline{\sqrt{\bar{w}}} \text { and } \sqrt[4]{w}=\overline{\sqrt[4]{\bar{w}}} \text {. }
$$

THEOREM 4. For $D$ a domain in $\Sigma_{n}$ which contains the point at infinity, the Szegö kernel function and its adjoint are, respectively, the functions

$$
\hat{K}(z, \bar{\zeta})=\frac{1}{4 \pi(z-\bar{\zeta})} \frac{\sqrt{m(z)}-\sqrt{m(\bar{\zeta})}}{\sqrt[4]{m(z)} \sqrt[4]{m(\bar{\zeta})}}
$$

and

$$
\hat{L}(z, \zeta)=\frac{1}{4 \pi(z-\zeta)} \frac{\sqrt{m(z)}+\sqrt{m(\zeta)}}{\sqrt[4]{m(z)} \sqrt[4]{m(\zeta)}}
$$

Proof. For $\zeta$ fixed in $D$, define functions $f_{1}(z)$ and $f_{2}(z)$ on $D$ by

$$
f_{1}(z)=\frac{1}{4 \pi(z-\bar{\zeta})} \frac{\sqrt{m(z)}-\sqrt{m(\bar{\zeta})}}{\sqrt[4]{m(z)} \sqrt[4]{m(\bar{\zeta})}}
$$

and

$$
f_{2}(z)=\frac{1}{4 \pi(z-\zeta)} \frac{\sqrt{m(z)}+\sqrt{m(\zeta)}}{\sqrt[4]{m(z)} \sqrt[4]{m(\zeta)}} ;
$$

$f_{1}(z)$ is analytic for all $z$ in $D$, while $f_{2}(z)$ is analytic for $z$ in $D$ except at the point $z=\zeta$ where it has a simple pole with residue $1 / 2 \pi$. For $z$ taken along the boundary of $D$, we claim that

$$
f_{2}(z) d z=i \overline{f_{1}(z)}|d z| .
$$

First suppose that $z$ is on the upper edge of one of the boundary segments of $D$. Then $d z=d x=|d z|, \sqrt{m(z)}=-\sqrt{m(z)}$, and for some $r>0$,

We thus have

$$
\overline{\sqrt[4]{m(z)}}=\mathrm{re}^{\overline{i \pi} / 4}=\mathrm{re}^{-i \pi / 4}=-i \sqrt[4]{m(z)} \text {. }
$$




$$
i \overline{f_{1}(z)}|d z|=\frac{1}{4 \pi(z-\zeta)} \frac{-\sqrt{m(z)}-\sqrt{m(\zeta)}}{-\sqrt[4]{m(z)} \sqrt[4]{m(\zeta)}} d z=f_{2}(z) d z
$$

as claimed. If $z$ is taken on the lower edge of one of the boundary segments, then $d z=-d x=-|d z|, \sqrt{\frac{m(z)}{2}}=-\sqrt{m(z)}$, and for some $r>0$,

$$
\sqrt[4]{m(z)}=\mathrm{re}^{\overline{-\pi i / 4}}=\mathrm{re}^{\pi i / 4}=i \sqrt[4]{m(z)}
$$

Thus,

$$
i \overline{f_{1}(z)}|d z|=\frac{1}{4 \pi(z-\zeta)} \frac{-\sqrt{m(z)}-\sqrt{m(\zeta)}}{\sqrt[4]{m(z)} \sqrt[4]{m(\zeta)}} d x=f_{2}(z) d z,
$$

and so equation (19) holds in either case. The defining conditions for the Szegö kernel function and its adjoint are thus satisfied, and the proof is complete.

The kernel functions $\hat{K}(z, \bar{\zeta})$ and $\hat{L}(z, \zeta)$ play an especially important role in the theory of bounded analytic functions. If we define

$$
B[D]=\{\text { functions } f(z) \text { analytic in } D:|f(z)|<1 \text { for } z \in D\},
$$

then the function

$$
\hat{F}(z, \zeta)=\hat{K}(z, \bar{\zeta}) / \hat{L}(z, \zeta)
$$

when viewed as an analytic function in the variable $z$, is a member of $B[D]$. Additionally, $\hat{F}(z, \zeta)$ solves the extremal problem $\left|f^{\prime}(\zeta)\right|=\max$, all $f \in B[D]$, and if $f_{0}(z)$ is a second extremal function, then for some real $\theta$,

$$
f_{0}(z)=e^{i \theta} \hat{F}(z, \zeta) .
$$

See, for example, [4, Chapter VII] and [7]. Compare also the original treatment due to Ahlfors [1] and that of Garabedian [5]. Observe that, for $D \in \Sigma_{n}$, we have

$$
\hat{F}(z, \zeta)=\frac{z-\zeta}{z-\bar{\zeta}} \cdot \frac{\sqrt{m(z)}-\sqrt{m(\bar{\zeta})}}{\sqrt{m(z)}+\sqrt{m(\zeta)}} \cdot \frac{\sqrt[4]{m(\zeta)}}{\sqrt[4]{m(\bar{\zeta})}},
$$

and thus the following theorem is immediate.

THEOREM 5. Let $D$ be a domain in $\Sigma_{n}$ which contains the point at infinity, and let $f(z)$ be analytic on $D$ and satisfy $|f(z)| \leqslant 1$. Then for all $z$ in $D$,

$$
\left|f^{\prime}(z)\right| \leqslant \begin{cases}\frac{1}{2 \sqrt{|m(z)|}} \frac{\operatorname{Im} \sqrt{m(z)}}{\operatorname{Im} z}, & \operatorname{Im} z \neq 0, \\ m^{\prime}(z) / 4 m(z), & \operatorname{Im} z=0,\end{cases}
$$


with equality at some point $z_{0}$ if and only if $f(z)=e^{i \theta} \hat{F}\left(z, z_{0}\right)$.

As a final application of Theorem 4 , let $D \in \Sigma_{n}$ have boundary components $\gamma_{0}, \gamma_{1}, \ldots, \gamma_{n-1}$ and induction coefficients $P_{\mu \nu}, \mu, \nu=1,2, \ldots, n-$ 1. The theta function (associated with the domain $D$ ) is then defined by

$$
\theta\left(z_{1}, z_{2}, \ldots, z_{n-1}\right)=\sum_{m} \exp \left\{-\pi^{2} \sum_{\mu, \nu} P_{\mu \nu} m_{\mu} m_{\nu}+2 \sum_{\mu} m_{\mu} z_{\mu}\right\}
$$

where $m=\left(m_{1}, \ldots, m_{n-1}\right)$ runs over all integral $(n-1)$-tuples. At the origin, $\theta$ has the expansion

$$
\theta\left(z_{1}, \ldots, z_{n-1}\right)=\theta(0)+\frac{1}{2} \sum_{j, k} \theta_{j, k}(0) z_{j} z_{k}+\ldots
$$

With this, we then have

$$
\begin{gathered}
4 \pi \hat{K}(z, \bar{\zeta})^{2}=K(z, \bar{\zeta})-\frac{\pi}{4} \sum_{j, k} \frac{\theta_{j k}(0)}{\theta(0)} w_{j}^{\prime}(z) \overline{w_{k}^{\prime}(\zeta)} \\
4 \pi \hat{L}(z, \zeta)^{2}=L(z, \zeta)-\frac{\pi}{4} \sum_{j, k} \frac{\theta_{j k}(0)}{\theta(0)} w_{j}^{\prime}(z) w_{k}^{\prime}(\zeta) .
\end{gathered}
$$

See [8].

Combining equations (14) and (21), we now obtain the following beautifully symmetric representations for the kernel functions $K(z, \zeta)$ and $L(z, \zeta)$ :

$$
\begin{aligned}
K(z, \zeta)= & \frac{1}{4 \pi}\left[\frac{\sqrt{q_{2}(z)} \sqrt{q_{1}(\zeta)}-\sqrt{q_{1}(z)} \sqrt{q_{2}(\zeta)}}{(z-\zeta)}\right]^{2} \frac{1}{\sqrt{q(z)} \sqrt{q(\zeta)}} \\
& +\frac{\pi}{4} \sum_{j, k} \frac{\theta_{j k}(0)}{\theta(0)} w_{j}^{\prime}(z) w_{k}^{\prime}(\zeta),
\end{aligned}
$$

and

$$
\begin{aligned}
L(z, \zeta)= & \frac{1}{4 \pi}\left[\frac{\sqrt{q_{2}(z)} \sqrt{q_{1}(\zeta)}+\sqrt{q_{1}(z)} \sqrt{q_{2}(\zeta)}}{(z-\zeta)}\right]^{2} \frac{1}{\sqrt{q(z)} \sqrt{q(\zeta)}} \\
& +\frac{\pi}{4} \sum_{j, k} \frac{\theta_{j k}(0)}{\theta(0)} w_{j}^{\prime}(z) w_{k}^{\prime}(\zeta) .
\end{aligned}
$$

Observe that we have reduced substantially the number of transcendentally defined kernel functions. On a domain in $\Sigma_{n}$ both the Szegö kernel function and its adjoint are elementary while the Bergman kernel function and its adjoint are essentially the same. Moreover, if the theta function and the differentials $w_{\mu}^{\prime}(z)$ are known, equations (22) and (23) completely identify the 
Bergman kernel function and its adjoint.

We remark that an alternative approach to the Szegö kernel function is through the Klein prime function. Indeed, Hejhal [8] was able to establish, in the case of an arbitrary Riemann surface, explicit formulas for the Szegö kernel function involving only the Klein prime function and the theta function. It is of particular interest to note here that, in the hyperelliptic case, the theta function can be written in terms of the Klein prime function, which in turn can be expressed in terms of the algebraic functions on the Riemann surface and their integrals. See, for example, Baker [2, pp. 433-466, 425-430]. Thus, in view of equations (22) and (23) above, it is possible to express the Bergman kernel function and its adjoint on a domain in the class $\Sigma_{n}$ entirely in terms of algebraic expressions.

\section{BIBLIOGRAPHY}

1. L. V Ahlfors, Bounded analytic functions, Duke Math. J. 14 (1947), 1-11. MR 9, 24.

2. H. F. Baker, Abel's theorem and the Allud theory, including the theory of theta functions, Cambridge Univ. Press, London and New York, 1897.

3. W. H. Barker, Plane domains with hyperelliptic double, Dissertation, Stanford Univ., 1975.

4. S. Bergman, The kernel function and conformal mappings, 2 nd ed., Math. Survey, no. 5, Amer. Math. Soc., Providence, R. I., 1970. (1st ed., 1950; MR 12, 402.)

5. P. R. Garabedian, Schwarz's lemma and the Szegö kernel function, Trans. Amer. Math. Soc. 67 (1949), 1-35. MR 11, 340.

6. N. S. Hawley and M. M. Schiffer, Half order differentials on Riemann surfaces, Acta Math. 115 (1966), 199-236. MR 32 \#739.

7. M. Heins, A lemma on positive harmonic functions, Ann. of Math. (2) 52 (1950), 568-573. MR 12, 259.

8. D. A. Hejhal, Theta functions, kernel functions, and Abelian differentials, Mem Amer. Math. Soc., no. 129, 1972. MR 51 \#8403.

9. Z. Nehari, Conformal mapping, McGraw-Hill, New York, 1952. MR 13, 640.

10. M. M. Schiffer, Various types of orthogonalization, Duke Math. J. 17 (1950), 329-366. MR $12,491$.

11. M. M. Schiffer and N. S. Hawley, Connections and conformal mappings, Acta Math. 107 (1962), 175-274. MR 32 \#202.

12. M. M. Schiffer and D. C. Spencer, Functionals of finite Riemann surfaces, Princeton Univ. Press, Princeton, N. J., 1954. MR 16, 461.

13. F. Schottky, Über die konforme Abbildung mehrfach zusammenhängender ebener Flächen, CreHles J. 83 (1877), 300-351.

Department of Mathematics, Stanford University, Stanford, California 94305

Daniel H. Wagner Associates, Station Square One, Paoli, Pennsylvania 19301 (Current address) 